\title{
The Current Role of Postoperative Radiation Therapy in Salivary Duct Carcinoma of the Parotid Gland: A Case Series Report and Review of the Literature
}

\author{
Pinelopi Theopisti Memtsa ${ }^{\mathrm{a}, \mathrm{d}}$, Konstantia Loga ${ }^{\mathrm{b}}$, Ariadni Kyriakogiannakic, \\ Aggeliki Fotarellib, Ioannis Tzitzikas ${ }^{\mathrm{a}}$, Kyriaki Pisteuou-Gompaki ${ }^{\mathrm{a}}$
}

\begin{abstract}
Salivary duct carcinoma is a rare and highly malignant tumor of the salivary glands. It has poor prognosis, one of the worst amongst all salivary gland tumors, with high rates of local recurrence and metastasis. Histologically, it is very similar to the ductal carcinoma of the breast, hence its name. We describe five new cases of salivary duct carcinoma of the parotid gland and the therapeutic approach that was followed, concentrating on radiation therapy. Due to the aggressiveness of the tumor, many researchers have suggested that the therapeutic approach should also be aggressive in nature utilizing multiple modalities including surgery, radiation therapy, chemotherapy and hormone therapy.
\end{abstract}

Keywords: Salivary duct carcinoma; Radiation therapy; Parotid gland

\section{Introduction}

Salivary duct carcinoma is a rare tumor arising from the ductal epithelium of the salivary gland and accounting for $1-3 \%$ of all malignant salivary gland tumors $[1,2]$. It is characterized as highly aggressive because of the high probability of local and distant recurrence and tumor-related deaths. Surgery remains the primary treatment, yet because of the

Manuscript accepted for publication May 19, 2014

${ }^{a}$ Department of Radiation Oncology, AHEPA Hospital, Aristotle
University of Thessaloniki, Thessaloniki, Greece
${ }^{\mathrm{b}}$ Department of Clinical Oncology, Theageneio Cancer Hospital,
Thessaloniki, Greece
${ }^{\mathrm{c} D e p a r t m e n t ~ o f ~ R a d i a t i o n ~ O n c o l o g y, ~ A l e x a n d r a ~ G e n e r a l ~ H o s p i t a l ~ o f ~}$
Athens, Athens, Greece
${ }^{\mathrm{d} C o r r e s p o n d i n g ~ A u t h o r: ~ P i n e l o p i ~ T h e o p i s t i ~ M e m t s a, ~ D e p a r t m e n t ~ o f ~}$
Radiation Oncology, AHEPA Hospital, Aristotle University, Psarron
26, 54453, Thessaloniki, Greece. Email: pennymemtsa@hotmail.com

doi: http://dx.doi.org/10.14740/jmc1812w anatomical location it is often difficult to achieve excision within healthy margins, due to the need for preservation of the facial nerve whenever it is not infiltrated. Postoperative radiation therapy is indicated in case of positive resection margins, local lymph node metastasis and/or facial nerve paralysis. Chemotherapy can be used for metastatic forms of the disease [3].

\section{Case Report}

\section{Patients}

This is a retrospective study of five patients that were treated at our hospital between March and July of 2013. Five patients with a median age of 58 years (range 49 - 68 years) presented with a mass and constant pain in the parotid gland accompanied by a variety of clinical presentations such as inability to move one side of the face and swelling of the parotid gland. The patients were submitted to a carefully detailed physical examination and a thorough medical history, so as to conclude to a final differential diagnosis that included a malignant tumor of the parotid gland as well as a benign type. The patients underwent a computed tomography (CT) scan or MRI of the head and neck area which indicated that a malignant tumor was the most likely diagnosis. They underwent parotidectomy with preservation of the facial nerve and in some cases neck dissection, with no complications. The histology report in all of the cases showed salivary duct carcinoma of the parotid gland. Patients' characteristics are shown in Table 1. Due to the high risk of local recurrence, the patients underwent adjuvant radiation therapy with a linear accelerator, $6 \mathrm{MV}$ beam energy. They received a total dose of 60 Gy in 30 daily fractions given within 6 weeks. None of the patients received chemotherapy.

\section{Radiation therapy techniques}

The patients were immobilized lying supine with the neck slightly extended with an immobilization device, wore a thermoplastic mask, which was made prior to the treatmentplanning CT scan. The treatment-planning CT scan was per- 
Table 1. Patient Characteristics

\begin{tabular}{ll}
\hline Gender (male/female) & $2: 3$ \\
Age: median (range) & $58(49-68)$ \\
Site & Parotid gland \\
Pathological TNM stage & \\
$\quad$ T1/T2/T3 & $1 / 3 / 1$ \\
$\quad$ N0/N1/N2B & $3 / 0 / 2$ \\
pStage & \\
$\quad$ I/II/IVa & $1 / 2 / 2$ \\
Surgical margins status & \\
$\quad$ Positive/close/negative & $1 / 1 / 3$ \\
Perineural invasion (yes/no) & $2 / 3$ \\
Lymphovascular invasion (yes/no) & $2 / 3$ \\
Neck dissection (yes/no) & $2 / 3$ \\
\hline
\end{tabular}

formed with intravenous contrast so that the major vessels of the neck were easily visualized and thus helped the contouring of lymph nodes. The CT slice thickness was $0.5 \mathrm{~cm}$. Preoperative imaging and discussion with the surgeon was important in order to carefully design our treatment planning. Fusion of MRI and CT images was particularly helpful in the cases with perineural invasion and was used in three of five patients.

\section{Target volume definition}

No gross tumor volume (GTV) was defined, unless there was a microscopic residual disease, so the clinical target volume (CTV) was contoured as the sites of possible microscopic disease. Particular attention was given to the deep excision margin when it was likely to be close or involved if the facial nerve was preserved. In the cases where there were indications for prophylactic neck radiotherapy, the ipsilateral level Ib, II and III nodes were included to the treated volume according to the international guidelines and receive dose of 46 Gy [3]. In case of adjuvant neck radiotherapy after neck dissection, the lymph node levels that should be treated were included in the CTV and receive total dose of $60 \mathrm{~Gy}$.

\section{Normal tissue tolerance}

The inner ear, the contra lateral parotid gland and the spinal cord were contoured as organs at risk (OAR), as there is a risk of xerostomia and/or deafness if the tolerance dose is exceeded. We managed to keep the mean dose of the inner ear $\leq 35$ Gy to minimize the risk of sensor neural hearing loss, and the mean dose of the lateral parotid gland $\leq 20$ Gy to minimize the xerostomia. The only adverse effect present in our patients was xerostomia.

\section{Results}

All the treatments were carried out without any adverse effects, apart from xerostomia which occurred in all patients. The follow-up is every 3 months for the first 2 years and all of the patients were educated about possible signs and symptoms of tumor recurrence, including hoarseness, pain, dysphagia, bleeding and enlarged lymph nodes. Recent followup of the patients at 6 months post-external beam radiation therapy revealed no signs of recurrence of the disease, while the xerostomia insisted in the same degree.

\section{Discussion}

Carcinomas of the salivary glands are rare tumors, which account for less than $1 \%$ of all head and neck malignancies. Most salivary gland tumors are benign and arise from the parotid glands [4]. The causal factors of these malignant tumors are unknown. The most common malignant tumor of the parotid gland is the mucoepidermoid carcinoma, while salivary duct carcinomas are rare but very aggressive in nature. They have an incidence of $1-3 \%$ of all malignant salivary gland tumors; they are located most commonly in the parotid gland and constitute $0.9-6 \%$ of all parotid's tumors [5].

Parotid malignancies present usually as asymptomatic masses, but as the lesions progress in size, clinical presentations can vary from a constant pain to inability to move one side of the face or one side of the tongue or even the shoul- 
der. Salivary duct carcinoma is a rapidly growing mass that presents with facial paralysis in about $40-60 \%$ of the time and lymphadenopathy in 35\% [5]. The functional status of the adjacent cranial nerves should be reported before starting any treatment. Salivary duct carcinoma has an increased risk for perineural invasion and high risk of lymph node metastasis (about $73 \%$ ) as well as perineural spread, so symptoms arising from this behavior, such as cervical masses, should be looked for [6]. Distant metastasis has been reported from $30 \%$ to $70 \%$, and most patients die of disseminated disease [7].

Imaging findings, such as CT scan and MRI play an important role in the diagnosis of the malignancy and its treatment. The malignant nature of the tumor is usually suggested by the infiltration of adjacent tissues. A tissue sample should be obtained in order to reach a more definite diagnosis. This can be done either by fine-needle aspiration, which is not always reliable, or by the surgical excision of the tumor [8]. The tumor usually is firm to the touch and may have a cystic component. When there is infiltration of adjacent tissues it is usually obvious.

Histologically salivary duct carcinomas share many characteristics with ductal carcinomas of the breast [5]. An amount of studies have described a constant over-expression of keratin, HER-2/neu, CEA and c-erd-B2, while there are studies that have also reported androgen receptors and prostate-specific antigen expression. Research in a molecular and biological basis is the most promising area for the determination of the development of malignant tumors of the parotid gland as well as for the prediction of their clinical course. Some studies stated that a combination of Her-2/neu expression with an elevated proliferation rate is associated with worst outcomes [5].

Local surgery should include total parotidectomy especially when there is facial paralysis present and neck dissection when it is necessary [8]. The aggressiveness of this tumor justifies adjuvant measures. When there is extra parotid extension, pathological resection margins or cervical lymph node metastasis postoperative radiation therapy should be considered [7].

Definitive radiation therapy should only be used in unresectable tumors, in cases of patients who are unfit for surgery, or when the patient refuses to undergo surgery. The local control rate of malignant parotid tumors with photon RT alone is approximately $30 \%$, and with fast neutron therapy is $60 \%$ [9]. Adjuvant external radiation therapy is indicated when risk of local recurrence is high, such as in cases of narrow (or invaded) surgical margins, perineural speed, lymphatic and/or vascular invasion, lymph-node involvement and high-grade histology [9]. The addition of radiotherapy can reduce local recurrence rates from $30 \%$ to $10 \%$ without any effect on overall survival [9]. The current radiotherapy techniques for the malignant tumors of the parotid gland include photon RT using 3D-CRT or IMRT and fast neutron radiotherapy.

A number of publications have been made so as to consolidate the role of the radiation therapy in the malignant tumors of the salivary glands, as well as the time that radiation therapy should take place regarding surgery and chemotherapy at which it should intervene. The Dutch Head and Neck Oncology Cooperative Group found that surgery without post-operative radiation therapy has a local failure relative risk of 9.7 (CI $4.4-21$ ), which is the highest relative risk for any factor that affects the local control [8]. A multivariate analysis that was performed found that the adjuvant radiation therapy decreased the risk of disease-specific death when compared to surgery alone and when it was adjusted for tumor size [10].

Other studies revealed that patients with malignant tumor of the parotid gland treated with surgery followed by adjuvant radiation therapy achieve superior recurrence-free survival at 10 years, while there was demonstrated an improved survival and local control in patients with locoregionally advanced diseases and a trend of improved outcome for high-grade diseases [11].

In the era of IMRT, the dose distribution within the target area is more homogeneous and we are able to minimize the dose to relevant normal structures. If IMRT technology is not available, we use optimal techniques such as three fields and mixed electron-photon beams so as to achieve the desirable homogenization of the dose [12].

Neutron radiotherapy is not available across the radiation-oncology centers and therefore the studies that have been done are limited range. However there is evidence of the effectiveness of this radiation therapy technique. Neutron radiotherapy has demonstrated higher rates of local control but increased risk of late toxicity. The increased risk of complications with neutron therapy, lack of availability and failure to demonstrate a survival benefit have hindered its success despite its superior local control over photons [9]. Radiation therapy of the head and neck area can have many adverse effects. Some of them include partial xerostomia, trismus, hearing loss, bone necrosis, cataract, dysfunction of the thyroid gland and retinopathy [4].

There has been no consensus regarding the role of chemotherapy to tumors of the salivary glands and this is because they show poor response to systemic therapy. A combination of doxorubicin, cyclophosphamide and cisplatin could be considered as an adjuvant therapy in addition to radiation therapy after surgery. However, systemic chemotherapy has a role in the treatment of distant disease and in recurrent salivary gland carcinoma [9]. The expression of HER-2/neu oncogene suggests that systemic therapy with trastuzumab could be indicated [13].

An amount of studies have shown that perineural and lymphovascular invasion is a significant prognostic factor in patients with parotid gland tumors. Surgery and postoperative radiotherapy are effective for locoregional control 
$[7,13]$. Surgery and postoperative radiotherapy decrease the risk of local recurrence and contribute to good clinical outcome [10]. Nevertheless, with the addition of radiation therapy, local recurrence and distant metastasis are frequent because of the aggressiveness of this specific tumor. Some studies suggest the irradiation field should include the skull base along with the cranial nerves that are involved, when perineural invasion coexists [7].

This study had several limitations. Some of them are the small number of patients (partially due to the rarity of this particular type of cancer) and the modest follow-up interval.

The management of salivary duct carcinomas should be multidisciplinary. Patients should be referred to experienced surgical Head and Neck Oncology Centers, and new treatment strategies should be investigated including the minimization of the adverse effects of radiation therapy, the role of chemotherapy and the role of hormone therapy.

\section{Conflict of Interest}

The authors declare no conflict of interest.

\section{References}

1. Salovaara E, Hakala O, Back L, Koivunen P, Saarilahti K, Passador-Santos F, Leivo I, et al. Management and outcome of salivary duct carcinoma in major salivary glands. Eur Arch Otorhinolaryngol. 2013;270(1):281285.

2. Colmenero Ruiz C, Patron Romero M, Martin Perez M. Salivary duct carcinoma: a report of nine cases. J Oral Maxillofac Surg. 1993;51(6):641-646.

3. Pons Y, Alves A, Clement P, Conessa C. Salivary duct carcinoma of the parotid. Eur Ann Otorhinolaryngol Head Neck Dis. 2011;128(4):194-196.

4. Terhaard CH. Salivary glands. In: Halperin EC, Perez CA, Brady LW, editors. Principles and Practice of Radiation Oncology, 5th Edition, Philadelphia: Lippincott Williams \& Wilkins; 2004. p. 407.

5. Jaehne M, Roeser K, Jaekel T, Schepers JD, Albert
$\mathrm{N}$, Loning T. Clinical and immunohistologic typing of salivary duct carcinoma: a report of 50 cases. Cancer. 2005;103(12):2526-2533.

6. Chong LM, Amstrong JG. Tumors of the salivary glands. In: Hoppe RT, Phillips TL, Roach M. editors. Leibel and Phillips Textbook of radiation oncology. 3rd Ed. NY: Elsevier; 2010. p. 666-700.

7. Locati LD, Bossi P, Licitra L. How many therapeutic options are there for recurrent or metastatic salivary duct carcinoma? J Clin Oncol. 2012;30(6):672; author reply 672-673.

8. Brandwein-gensler, Skalova A, Nagao T. Salivary duct carcinoma. In: Barnes L, Eveson JW, Sidransky D, editors. World Health Organization Classification of tumours, Pathology and genetics of head and neck tumours. Lyon: IARCC Press; 2003. p. 236-238.

9. Terhaard CH, Lubsen H, Van der Tweel I, Hilgers FJ, Eijkenboom WM, Marres HA, Tjho-Heslinga RE, et al. Salivary gland carcinoma: independent prognostic factors for locoregional control, distant metastases, and overall survival: results of the Dutch head and neck oncology cooperative group. Head Neck. 2004;26(8):681692; discussion 692-683.

10. Koul R, Dubey A, Butler J, Cooke AL, Abdoh A, Nason R. Prognostic factors depicting disease-specific survival in parotid-gland tumors. Int J Radiat Oncol Biol Phys. 2007;68(3):714-718.

11. Armstrong JG, Harrison LB, Spiro RH, Fass DE, Strong EW, Fuks ZY. Malignant tumors of major salivary gland origin. A matched-pair analysis of the role of combined surgery and postoperative radiotherapy. Arch Otolaryngol Head Neck Surg. 1990;116(3):290-293.

12. Yaparpalvi R, Fontenla DP, Tyerech SK, Boselli LR, Beitler JJ. Parotid gland tumors: a comparison of postoperative radiotherapy techniques using three dimensional (3D) dose distributions and dose-volume histograms (DVHS). Int J Radiat Oncol Biol Phys. 1998;40(1):4349.

13. Roh JL, Cho KJ, Kwon GY, Choi SH, Nam SY, Kim SY. Prognostic values of pathologic findings and hypoxia markers in 21 patients with salivary duct carcinoma. J Surg Oncol. 2008;97(7):596-600. 